\title{
Prognosis by novel staging systems incorporating tumor biology in estrogen receptor positive and human epidermal growth factor receptor negative breast tumors
}

Marissa Vane ( $\sim$ marissa.vane@gmail.com )

Maastricht Universitair Medisch Centrum+ https://orcid.org/0000-0003-4514-5329

Fenna Traa

Maastricht University Hospital: Maastricht Universitair Medisch Centrum+

Lori van Roozendaal

Maastricht University Hospital: Maastricht Universitair Medisch Centrum+

Sander van Kuijk

Maastricht University Hospital: Maastricht Universitair Medisch Centrum+

Sanne Engelen

Maastricht University Hospital: Maastricht Universitair Medisch Centrum+

Marissa van Maaren

IKNL: Integraal Kankercentrum Nederland

Sabine Siesling

Integraal Kankercentrum Nederland

Johannes de Wilt

Radboudumc

Marjolein Smidt

Maastricht University Hospital: Maastricht Universitair Medisch Centrum+

\section{Research Article}

Keywords: breast cancer staging system, Bioscore, 8th AJC TNM system, recurrence-free survival, ER+HER2- tumors

Posted Date: March 1st, 2022

DOI: https://doi.org/10.21203/rs.3.rs-1396504/v1

License: (9) This work is licensed under a Creative Commons Attribution 4.0 International License.

Read Full License 



\title{
Prognosis by novel staging systems incorporating tumor biology in estrogen receptor positive and human epidermal growth factor receptor negative breast tumors
}

\author{
M.L.G. Vane ${ }^{1,2}$, F. Traa ${ }^{1}$, L.M. van Roozendaal ${ }^{1}$, S.M.J. van Kuijk ${ }^{3}$, \\ S.M.E. Engelen ${ }^{1}$, M.C. van Maaren ${ }^{4,5}$, S. Siesling ${ }^{4,5}$, J.H.W. de Wilt ${ }^{6}$, M.L. Smidt ${ }^{1,2}$ \\ ${ }^{1}$ Department of Surgical Oncology, Maastricht University Medical Centre, Maastricht, the Netherlands. \\ ${ }^{2}$ GROW - School for Oncology and Developmental Biology, Maastricht University Medical Centre, the \\ Netherlands. \\ ${ }^{3}$ Department of Epidemiology, Maastricht University Medical Center+, Maastricht, The Netherlands. \\ ${ }^{4}$ Department of Research and Development, Netherlands Comprehensive Cancer Organisation (IKNL), \\ Utrecht, the Netherlands. \\ ${ }^{5}$ Department of Health Technology and Services Research, Faculty of Behavioural, Management and \\ Social Sciences, Technical Medical Centre, University of Twente, Enschede, the Netherlands. \\ ${ }^{6}$ Division of Surgical Oncology, Radboud University Medical Centre, Nijmegen, the Netherlands.
}

\section{Corresponding author}

Marissa Vane, MD

Department of Surgical Oncology

Maastricht University Medical Centre

P.O. Box 5800

6202 AZ Maastricht

The Netherlands

Phone: +31 433881574

Mail: marissa.vane@gmail.com 


\begin{abstract}
Purpose The prognostic stage group and Bioscore are novel staging systems for breast cancer patients. They provide a better view on five-year disease-specific survival (DSS) compared to the traditional staging system. Five-year follow-up might not be sufficient for ER+HER2tumors. This study determines five- and 10-year recurrence-free survival (RFS) for the anatomic and prognostic stage group of the $8^{\text {th }}$ AJCC TNM and Bioscore staging system, and compares these systems for ER+HER2- breast cancer patients.

Methods All newly diagnosed ER+HER2- breast cancer patients treated with primary surgery in 2005 were included from the Netherlands Cancer Registry with a ten-year follow-up. All patients were staged according to $8^{\text {th }}$ AJCC TNM (both anatomic and prognostic stage group) and the Bioscore staging system. Five- and 10-year RFS was compared using Kaplan-Meier analysis. The ability to discriminate groups was quantified by the concordance statistic (cstatistic).
\end{abstract}

Results Five-year RFS ranged from 65.0-91.1\% (anatomic) and from 40.9-91.8\% (prognostic stage group). The Bioscore staging system ranged from 54.9-92.1\%. Ten-year RFS rates were $52.9-84.9 \%$ (anatomic stage), 31.8-84.6\% (prognostic stage) and 47.1-85.1\% (Bioscore). All systems similarly discriminated groups with comparable c-statistic score (five- and 10-year $0.58-0.60)$. The prognostic stage group identified a group(IIIC) with very poor prognosis (31.8\%) which could not be identified using the other staging systems (10-year RFS for $52.8 \%$ for anatomic IIIC and $47.1 \%$ for Bioscore 6 ).

Conclusion All three staging systems similarly discriminated groups for ER+HER2- breast cancer patients with a five- and 10-year follow-up. However, the prognostic stage group showed a wider distribution of the 10-year RFS compared to anatomic stage group and Bioscore staging system.

KEYWORDS breast cancer staging system, Bioscore, 8th AJC TNM system, recurrence-free survival, ER+HER2- tumors 


\section{BACKGROUND}

Breast cancer is usually staged according to the TNM classification of the American Joint Committee on Cancer (AJCC) system. The anatomic stage group of the AJCC is based on tumor size $(\mathrm{T})$, lymph node status $(\mathrm{N})$, and presence or absence of distant metastasis $(\mathrm{M})$, and is used in breast cancer patients to assess their prognosis and guide their treatment plan (stage 0 to IV). However, tumor biology is not included in this staging system, resulting in a wide variation of prognosis within each TNM stage. Additionally, tumor biology expressed as grade and receptor status have become important prognostic and predictive factors in breast cancer patients (1-3).

The $8^{\text {th }}$ edition of the AJCC TNM staging system recognized the lack of tumor biology and introduced the prognostic stage group. In this staging system, the anatomic stage characteristics (TNM) are combined with tumor grade, estrogen receptor (ER), progesterone receptor (PR), and human epidermal growth factor receptor (HER2), and can even include the multigene status (e.g. Oncotype DX) if available. The prognostic stage group identifies several prognostic groups (stage 0 to IV) (4). Another novel staging system is called Bioscore and includes pathologic tumor size, lymph node status, and tumor biology in terms of tumor grade, ER and HER2 receptor status for patients primarily treated with surgery (score 0-7) $(5,6)$. So far, both staging systems are validated for breast cancer patients primarily treated with surgery with a median follow-up of five years (5-7). Although, ER+HER2- tumors have the lowest risk of recurrence of all breast cancer subtypes (10-12), late recurrences (after five years of diagnosis), continue to occur especially in this subgroup (13). Pan et al. showed that recurrences keep occurring in ER+ tumors, even up to 20 years after diagnosis. Hormone therapy in ER+ breast cancer patients potentially postpones these recurrences in several cases (14). Five-year follow-up is therefore not sufficient for ER+HER2- breast cancer patients.

The aim of this study was to determine and compare five- and 10-year recurrence-free survival (RFS) for the anatomic and the prognostic stage group of the $8^{\text {th }}$ edition of the AJCC TNM system and the Bioscore staging system, and to compare these systems for ER+HER2invasive breast cancer patients primarily treated with surgery. 


\section{METHODS}

Data were retrieved from the Netherlands Cancer Registry (NCR). The NCR contains data on patient demographics and information regarding the tumor as well as surgery, radiation, systemic treatment, recurrence and survival. Trained data registrars collected the information directly from patients' medical records in all Dutch hospitals, on the basis of a notification from the Pathological Anatomy National Automated Archive. All newly diagnosed ER+HER2- breast cancer patients in the Netherlands in 2005 were included if primarily treated with surgery. Patients were excluded in case of unknown data regarding tumor grade, pathologic tumor status, lymph node status or receptor status $(n=511)$. Tumors were classified ER+ if at least $10 \%$ of the nuclei stained. Tumors were classified HER2 negative if less than $10 \%$ of the cells showed membrane staining. In case of $2+$ at immunohistochemistry, an amplification test was performed that overruled the results of the immunohistochemistry.

\section{Endpoints}

The primary endpoint of this study was five- and 10-year RFS. RFS was defined as the date of surgery until the date of first breast cancer recurrence (local recurrence, regional recurrence, new primary ipsilateral breast cancer, contralateral breast cancer, or distant metastasis), death of any cause, or the date of last observation. Events within 91 days following diagnosis were regarded as synchronous events. Baseline characteristics were presented as mean or median and standard deviation or range, or absolute numbers and percentages.

First, all patients were staged according to the anatomic and prognostic stage group of the $8^{\text {th }}$ edition of the AJCC TNM staging system. The anatomic stage group is based on tumor size (T1-4), lymph node status (N0-3), and presence or absence of distant metastasis (M0-1). The prognostic stage group includes TNM status, tumor grade (I-III), ER status (positive/negative), PR status (positive/negative) and HER2 status (positive/negative) (9). For both stage groups, final staging score ranged from I to IV. Subsequently, patients were staged using the Bioscore staging system. Each tumor characteristic was given a score based on the hazard ratio for DSS: pathological T stage $(\mathrm{T} 1=0$ and $\mathrm{T} 2$ or $\mathrm{T} 3=1)$, pathological $\mathrm{N}$ stage $(\mathrm{N} 0, \mathrm{~N} 1 \mathrm{mic}$ or $\mathrm{N} 1=0, \mathrm{~N} 2=2$ and $\mathrm{N} 3=3)$, tumor grade $($ grade $\mathrm{I}-\mathrm{II}=0$ and grade $\mathrm{III}=1), \mathrm{ER}$ receptor status $(\mathrm{ER}+=0$ and $\mathrm{ER}-=1)$ and HER2 receptor status $(\mathrm{HER} 2+=0, \mathrm{HER} 2-=1)$. The Bioscore was calculated by the sum of each tumor characteristic, ranging from 0 to 7 points (7). 


\section{Statistical analyses}

Five- and 10-year RFS for the $8^{\text {th }}$ edition of the AJCC TNM system (anatomic and prognostic stage group separately) and Bioscore staging system were compared using Kaplan Meier analysis. The ability of these systems to discriminate groups based on risk of a recurrence was quantified by the concordance statistic (c-statistic) after fitting the staging systems separately in a Cox proportional hazards regression model. Statistical analyses were performed using the Statistical Package for the Social Sciences (SPSS), version 26.0 and R version 3.3.3.

\section{RESULTS}

In total, 4.229 ER+HER2- breast cancer patients primarily treated with surgery were included. Median follow-up was 9.6 years (range $0.1-12.0$ ). Patient and tumor characteristics are summarized in Table 1.

Using the anatomic stage group of the $8^{\text {th }}$ edition of the AJCC TNM staging system, 2.041 (48.3\%) patients were classified as stage I (A and B), followed by stage IIA in 1.209 (28.6\%), stage IIB in $499(11.8 \%)$, stage IIIA in $323(7.6 \%)$ and stage IIIC $157(3.7 \%)$ of the patients. None of the patients were staged with IIIB (T4N0-2M0) or stage IV tumors (M1). Using the prognostic stage group of the $8^{\text {th }}$ edition of the AJCC TNM staging system, 2.821 (66.7\%) patients were classified as stage I (A and B), followed by stage IIA in $815(19.3 \%)$, stage IIB in $254(6.0 \%)$, stage IIIA in $154(3.6 \%)$, stage IIIB in $163(3.9 \%)$ and stage IIIC in $22(0.5 \%)$ of the patients.

Using the Bioscore staging system, 2.179 (51.5\%) scored 1 point, $1.222(28.9 \%)$ scored 2 points, $453(10.7 \%)$ scored 3 points, 169 (4.0\%) scored 4 points, $155(3.7 \%)$ scored 5 points and $51(1.2 \%)$ scored 6 points. None of the patients scored 0 points or 7 points, since only ER+HER2- patients were included in this study (Table 2).

Five-year RFS was $88.6 \%$ in the overall population. Using the anatomic stage group of the $8^{\text {th }}$ edition of the AJCC TNM staging system, RFS ranged from 65.0 to $91.1 \%$ for the subgroups (Table 2). For the prognostic stage group, five-year RFS ranged from 40.9 to $91.8 \%$. Using the Bioscore staging system, RFS ranged from 54.9 to $92.1 \%$ for the subgroups (Table 2). Ten-year RFS was $80.6 \%$ in the overall population. Using the anatomic stage group of the $8^{\text {th }}$ edition of the AJCC TNM staging system, RFS ranged from 52.9 to $84.9 \%$. For stage I, IIA, IIB, IIIA and IIIC, this was 83.4\%, 84.9\%, 75.2\%, 68.4\% and 52.9\%, respectively (Table 2). Using the prognostic stage group of the $8^{\text {th }}$ edition of the AJCC TNM staging system, survival 
ranged from 31.8 to $84.6 \%$. For stage I, IIA, IIB, IIIA and IIIC, this ranged from $84.6 \%$ to $31.8 \%$, respectively (Table 2). Using the Bioscore staging system, 10-year RFS ranged from $47.1 \%$ to $85.1 \%$. For Bioscore 1, 2, 3, 4, 5 and 6 this ranged from $85.1 \%$ to $47.1 \%$, respectively (Table 2, Figure $2 \mathrm{~B}$ ).

The c-statistic score for the anatomic and prognostic group of the $8^{\text {th }}$ AJCC was 0.58 and 0.59 compared to 0.59 using the Bioscore staging system, in a five-year follow-up period and 0.58 , 0.60 and 0.60 in a 10 -year follow-up period. 


\section{DISCUSSION}

This is the first study comparing the anatomic and prognostic stage group of the $8^{\text {th }}$ edition of the AJCC TNM system and the Bioscore staging system for ER+HER2- invasive breast cancer patients primarily treated with surgery using a ten-year follow-up period. Five-year RFS using the anatomic stage group of the $8^{\text {th }}$ edition of the AJCC TNM system ranged from $65.0-91.1 \%$, using the prognostic stage group this ranged from $40.9-91.8 \%$ and $54.9-92.1 \%$ using the Bioscore staging system. Ten-year RFS ranged from 52.9-83.4\%, 31.8-84.6\% and $47.1-85.1 \%$, respectively. All systems similarly discriminated groups according to the risk of recurrence, with comparable c-statistic score at five and 10-years. The prognostic stage group of the $8^{\text {th }}$ AJCC TNM system showed a wider distribution of the 10-year RFS compared to anatomic stage group and Bioscore staging system.

To address the importance of tumor biology, the prognostic stage group was introduced in the $8^{\text {th }}$ edition of the AJCC TNM staging in addition to the anatomic stage group $(4,9,16,17)$. Weiss et al. validated the prognostic stage group using a cohort of 3.327 breast cancer patients primarily treated with surgery diagnosed between 2007-2013. Subsequently, this stage group was validated using the California Cancer Registry ( $n=54.727$ stage I-IV breast cancer patients). Results showed that the prognostic stage group upstaged 31.0\% and downstaged $20.6 \%$ of the included patients. Furthermore, the prognostic stage group provided a more accurate five-year DSS stratification compared to anatomic stage group (c-statistic 0.84 versus 0.81 ) (4). These results were confirmed by this study showing a wider distribution of 10-year RFS ranging from 31.8 to $84.6 \%$ using the prognostic stage group compared to 52.9 to $84.9 \%$ using the anatomic stage group in ER+HER2- breast cancer patients. An advantage of the prognostic stage group is the incorporation of the gene expression profile Oncotype DX, predicting tumor recurrence and helping to stratify patients who will benefit from chemotherapy in ER + HER2- tumors (4). The Oncotype DX was in this study not available and therefore not included, similar to the study of Weiss et al. Disadvantages of the prognostic stage group are the 170 different groups, making this staging system clinically complex to use (4). Furthermore, some countries do not have access to analysis all these biological factors. For those, the anatomic staging group of the $8^{\text {th }}$ edition of the AJCC TNM system remains recommended (16). 
The Bioscore staging system was developed by MD Anderson using the same cohort of 3.327 breast cancer patients as Weiss et al. Several models with different combinations of tumor biology were investigated, and ultimately the Bioscore staging system including anatomic stage, tumor grade, ER and HER2 status was developed. The Bioscore staging system was validated using the California Cancer Registry ( $n=67.944$ patients). Five-year DSS ranged from $33.3 \%$ to $100 \%$ for different tumor stages using Bioscore, compared to $79.5 \%$ to $99.1 \%$ using the anatomic stage group of the $7^{\text {th }}$ AJCC TNM staging system $(6,7)$. This present study showed a range of 10-year RFS from 47.1 to $85.1 \%$ using the Bioscore staging system compared to 52.9 to $84.9 \%$ using the anatomic stage group of the $8^{\text {th }}$ edition of the AJCC TNM staging system in ER+HER2- breast cancer patients. Mittendorf showed that improved stratification was most pronounced in patients with higher Bioscores (five-year DSS of 33.3\% for Bioscore 7 versus $79.5 \%$ for stage IIIC). This study showed that patients with higher Bioscore had the lowest 10-year RFS (10-year RFS 47.1\% for Bioscore 6. However, the prognostic stage group of the AJCC TNM system identified a group of patients with a worse prognosis (stage IIIC, 10-year RFS 31.8\%) which could not be identified using the anatomic stage group (stage IIIC, 52.9\%) nor Bioscore staging system (Bioscore 6, 10-year RFS $47.1 \%)$. The c-statistic score for ten-year RFS for anatomic and prognostic stage group were 0.58 and 0.60 versus 0.60 for Bioscore staging system, implying that staging systems similarly discriminated groups according to the risk of recurrence. This is in contrast to the study of Mittendorf et al. who showed that the Bioscore had a higher c-statistic score (0.81), however this was compared to the $7^{\text {th }}$ AJCC staging system (0.74) and using a five-year follow-up period (6).

A strength of this study is the large population-based registry of 4.229 ER+HER2- breast cancer patients with complete data on tumor staging and biology and a ten-year follow-up. Subgroups of the staging systems in this present study were not equally divided, especially for higher stage disease which is in line with the population incidence. There were only limited patient numbers for stage IIIA, IIIB and IIIC (7.6\%, 0\% and 3.7\%, respectively) using the anatomic stage group and the prognostic stage group (3.6\%, 3.9\% and $0.5 \%$, respectively). Subgroups according to Bioscore also revealed low numbers for higher scores 4 to 7 (4.0\%, $3.7 \%, 1.2 \%$, and $0 \%$ respectively). This was similar for the cohort of Mittendorf et al., with $0.3 \%$ of the patients with lowest DSS of $33.4 \%$ with a Bioscore of 7 , and highest DSS of $100 \%$ in $1.1 \%$ of the patients with a Bioscore of 0 . Higher stage disease patients are often treated with neoadjuvant systemic therapy and therefore not included in these studies. For this 
subset of patients, the Neo-Bioscore staging system was developed $(6,7,18)$. While interpreting the current results, it has to be kept in mind that the lower and upper limits of both staging systems are determined by a small subgroup of the overall population.

Due to several differences between the studies, results are difficult to compare.

First, this study used RFS instead of DSS used by Mittendorf and Weis at al. (7, 4), as data on DSS were not available in the NCR database. Breast cancer recurrence occurs more often than death from breast cancer (DSS), and therefore explains the lower rates of RFS in this present study (five-year RFS of $88.6 \%$ and 10-year RFS of 80.6\%) compared to five-year DSS of $97.9 \%$ in the study o Mittendorf et al.

Another difference between the studies was the number of patients treated with adjuvant systemic therapy caused by a different recommendation for adjuvant systemic therapy. Adjuvant systemic therapy was recommended only for patients $<35$ years and for patients $\geq 35$ years with risk factors (i.e. tumor $\geq 3 \mathrm{~cm}$, or tumor $\geq 1 \mathrm{~cm}$ and grade III, or tumor $\geq 2 \mathrm{~cm}$ and grade II) (15). As a result, only 55.9\% of the ER+HER2- patients in this study were treated with HT, whereas all ER+ patients in the study of Mittendorf et al. were advised and $81.2 \%$ were treated with HT. Chemotherapy was given in $32.1 \%$ in this study compared to $49.2 \%$ of the patients from MD Anderson. Since none of the tumors was HER2+ (since it was an exclusion criterium), no indication for trastuzumab existed compared to $9.2 \%$ of the patients in the MD Anderson cohort (HER2+ tumors). Kantor et al. compared the prognostic stage group, Bioscore staging system and Risk Score in a large population-based cohort using Surveillance, Epidemiology and End Results program (SEER). Results showed that the cstatistic of each novel staging system was higher than the c-statistic for the anatomic stage group of the AJC TNM staging system (c-statistic 0.83 anatomic stage group versus 0.86 for the prognostic stage group, 0.86 for the Bioscore staging system, and 0.86 for the Risk Score, $\mathrm{p}<0.001)(19)$.

In conclusion, this study aimed to determine five- and 10-year RFS for the anatomic stage group and the novel prognostic stage group of the $8^{\text {th }}$ edition of the AJCC TNM system and the Bioscore staging system for ER+HER2- breast cancer patients with a ten-year follow-up. All three staging systems similarly discriminated groups for ER+HER2- breast cancer patients with a 10-year follow-up. The prognostic stage group of the $8^{\text {th }}$ AJCC TNM system showed a wider distribution of the 10-year RFS compared to anatomic stage group and Bioscore staging 
system. Therefore, this staging system identified a group with a very poor prognosis $(31.8 \%)$ which could not be identified using the anatomic stage group of the $8^{\text {th }}$ AJCC (stage IIIC, $52.8 \%$ ) or Bioscore staging system (Bioscore 6, 47.1\%). We would like to suggest that hospitals who have access to analysis of biological factors, use the prognostic stage group in addition to the traditional anatomic stage group of the $8^{\text {th }}$ AJCC TNM staging system to determine a more patient-tailored prognosis. 


\section{REFERENCES}

1. Van Poznak C, Harris LN, Somerfield MR. Use of Biomarkers to Guide Decisions on Systemic Therapy for Women With Metastatic Breast Cancer: American Society of Clinical Oncology Clinical Practice Guideline. Journal of oncology practice / American Society of Clinical Oncology. 2015;11(6):514-6.

2. Harris LN, Ismaila N, McShane LM, Andre F, Collyar DE, Gonzalez-Angulo AM, et al. Use of Biomarkers to Guide Decisions on Adjuvant Systemic Therapy for Women With Early-Stage Invasive Breast Cancer: American Society of Clinical Oncology Clinical Practice Guideline. Journal of clinical oncology : official journal of the American Society of Clinical Oncology. 2016;34(10):1134-50.

3. Coates AS, Winer EP, Goldhirsch A, Gelber RD, Gnant M, Piccart-Gebhart M, et al. Tailoring therapies--improving the management of early breast cancer: St Gallen International Expert Consensus on the Primary Therapy of Early Breast Cancer 2015. Annals of oncology : official journal of the European Society for Medical Oncology / ESMO. 2015;26(8):1533-46.

4. Weiss A, Chavez-MacGregor M, Lichtensztajn DY, Yi M, Tadros A, Hortobagyi GN, et al. Validation Study of the American Joint Committee on Cancer Eighth Edition Prognostic Stage Compared With the Anatomic Stage in Breast Cancer. JAMA oncology. 2018;4(2):203-9

5 Yi M, Mittendorf EA, Cormier JN, Buchholz TA, Bilimoria K, Sahin AA, et al. Novel staging system for predicting disease-specific survival in patients with breast cancer treated with surgery as the first intervention: time to modify the current American Joint Committee on Cancer staging system. Journal of clinical oncology : official journal of the American Society of Clinical Oncology. 2011;29(35):4654-61.

6. Mittendorf EA, Jeruss JS, Tucker SL, Kolli A, Newman LA, Gonzalez-Angulo AM, et al. Validation of a novel staging system for disease-specific survival in patients with breast cancer treated with neoadjuvant chemotherapy. Journal of clinical oncology : official journal of the American Society of Clinical Oncology. 2011;29(15):1956-62.

7. Mittendorf EA, Chavez-MacGregor M, Vila J, Yi M, Lichtensztajn DY, Clarke CA, et al. Bioscore: A Staging System for Breast Cancer Patients that Reflects the Prognostic Significance of Underlying Tumor Biology. Annals of surgical oncology. 2017;24(12):3502-9.

8. Donovan CA, Giuliano AE. Evolution of the Staging System in Breast Cancer. Annals of surgical oncology. 2017;24(12):3469-70.

9. Amin MB, Greene FL, Edge SB, Compton CC, Gershenwald JE, Brookland RK, et al. The Eighth Edition AJCC Cancer Staging Manual: Continuing to build a bridge from a population-based to a more "personalized" approach to cancer staging. CA: a cancer journal for clinicians. 2017;67(2):93-9.

10. Lowery AJ, Kell MR, Glynn RW, Kerin MJ, Sweeney KJ. Locoregional recurrence after breast cancer surgery: a systematic review by receptor phenotype. Breast cancer research and treatment. 2012;133(3):831-41. 11. van Maaren MC, de Munck L, Strobbe LJA, Sonke GS, Westenend PJ, Smidt ML, et al. Ten-year recurrence rates for breast cancer subtypes in the Netherlands: A large population-based study. International journal of cancer Journal international du cancer. 2019;144(2):263-72.

12. Voduc KD, Cheang MC, Tyldesley S, Gelmon K, Nielsen TO, Kennecke H. Breast cancer subtypes and the risk of local and regional relapse. Journal of clinical oncology : official journal of the American Society of Clinical Oncology. 2010;28(10):1684-91. 
13. Matsen C, Villegas K, Eaton A, Stempel M, Manning A, Cody HS, et al. Late Axillary Recurrence After Negative Sentinel Lymph Node Biopsy is Uncommon. Annals of surgical oncology. 2016;23(8):2456-61. 14. Pan H, Gray R, Braybrooke J, Davies C, Taylor C, McGale P, et al. 20-Year Risks of Breast-Cancer Recurrence after Stopping Endocrine Therapy at 5 Years. The New England journal of medicine. 2017;377(19):1836-46.

15. Guideline Mammacarcinoma the Netherlands the Netherlands. Available from: http://richtlijnendatabase.nl/en/richtlijn/breast_cancer/locoregional_treatment.html.2005 16. Giuliano AE, Connolly JL, Edge SB, Mittendorf EA, Rugo HS, Solin LJ, et al. Breast Cancer-Major changes in the American Joint Committee on Cancer eighth edition cancer staging manual. CA: a cancer journal for clinicians. 2017;67(4):290-303.

17. Chavez-MacGregor M, Mittendorf EA, Clarke CA, Lichtensztajn DY, Hunt KK, Giordano SH. Incorporating Tumor Characteristics to the American Joint Committee on Cancer Breast Cancer Staging System. The oncologist. 2017;22(11):1292-300.

18. Mittendorf EA, Vila J, Tucker SL, Chavez-MacGregor M, Smith BD, Symmans WF, et al. The NeoBioscore Update for Staging Breast Cancer Treated With Neoadjuvant Chemotherapy: Incorporation of Prognostic Biologic Factors Into Staging After Treatment. JAMA oncology. 2016;2(7):929-36.

19. Kantor, O., Niu, J., Zhao, H. et al. Comparative Analysis of Proposed Strategies for Incorporating Biologic Factors into Breast Cancer Staging. Ann Surg Oncol 27, 2229-2237 (2020) 


\section{STATEMENTS AND DECLARATIONS}

\section{Funding}

The authors declare that no funds, grants, or other support were received during the preparation of this manuscript.

\section{Competing interests}

M.L. Smidt, has been awarded a grant from Servier Pharma for microbiome research in breast cancer treatment. The remaining authors have no disclosures or conflicts of interest.

\section{Author contributions}

All authors contributed to the study conception and design. Material preparation, data collection and analysis were performed by M. Vane and F. Traa, M. van Maaren, S. Siesling, M. Smidt. The first draft of the manuscript was written by M. Vane and F. Traa and all authors commented on previous versions of the manuscript. All authors read and approved the final manuscript.

\section{Data availability}

The datasets were analysed during the current study are not publicly available due but are available from the corresponding author on reasonable request.

\section{Ethics approval}

The study was performed in accordance with the ethical standards as laid down in the 1964 Declaration of Helsinki and its later amendments or comparable ethical standards. Due to its retrospective design, ethical approval was not obtained.

\section{Consent to participate}

Not applicable.

\section{Acknowledgements}

The authors thank the registration team of the Netherlands Comprehensive Cancer Organisation (IKNL) for the collection of data for the Netherlands Cancer Registry. 


\section{Supplementary Files}

This is a list of supplementary files associated with this preprint. Click to download.

- NovelstagingsystemsTable1.pdf

- NovelstagingsystemsTable2.pdf 